\section{$\gamma$-Secretase Activity Is Associated with Braak Senile Plaque Stages}

Nobuto Kakuda, ${ }^{* \dagger+}$ Haruyasu Yamaguchi, ${ }^{\S}$ Kohei Akazawa, "Saori Hata, "Toshiharu Suzuki, Hiroyuki Hatsuta, ** Shigeo Murayama, ${ }^{* *}$ Satoru Funamoto, ${ }^{* \dagger}$ and Yasuo Ihara ${ }^{* \dagger+\dagger \dagger}$

From the Department of Neuropathology, ${ }^{*}$ the Center for Research in Neurodegenerative Diseases, ${ }^{\dagger}$ and the Graduate School of Brain Science and Faculty of Life and Medical Sciences, ${ }^{\dagger \dagger}$ Doshisha University, Kyoto; the Core Research for Evolutional Science and Technology, ${ }^{\ddagger}$ Japan Science and Technology Agency, Tokyo; the Faculty of Medicine School of Health Sciences, ${ }^{\S}$ Gunma University, Maebashi; the Department of Medical Informatics, ${ }^{\boldsymbol{\top}}$ Niigata University Medical and Dental Hospital, Niigata University, Niigata; the Department of Neuropathology," Tokyo Metropolitan Institute of Gerontology, Tokyo; and the Laboratory of Neuroscience, Graduate School of Pharmaceutical Sciences, ** Hokkaido University, Hokkaido, Japan

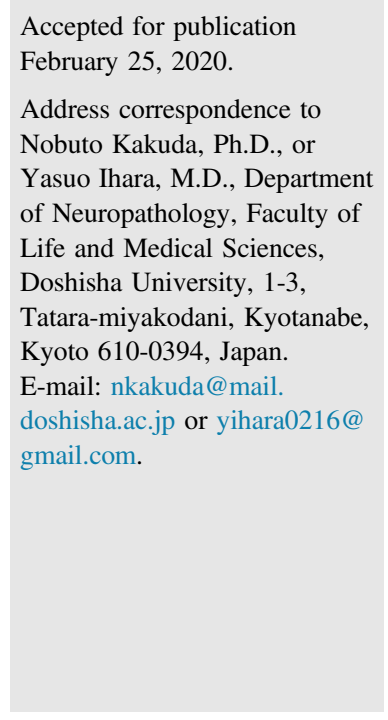

Accepted for publication

February 25, 2020

Address correspondence to Nobuto Kakuda, Ph.D., or Yasuo Ihara, M.D., Department of Neuropathology, Faculty of Life and Medical Sciences, Doshisha University, 1-3, Tatara-miyakodani, Kyotanabe, Kyoto 610-0394, Japan E-mail: nkakuda@mail. doshisha.ac.jp or yihara0216@ gmail.com. Check for updates
Amyloid $\beta$-proteins $(A \beta s) A \beta 1-42$ and $A \beta 1-43$ are converted via two product lines of $\gamma$-secretase to $A \beta 1-38$ and $A \beta 1-40$. This parallel stepwise processing model of $\gamma$-secretase predicts that $A \beta 1-42$ and $A \beta 1-43$, and $A \beta 1-38$ and $A \beta 1-40$ are proportional to each other, respectively. To obtain further insight into the mechanisms of parenchymal $A \beta$ deposition, these four $A \beta$ species were quantified in insoluble fractions of human brains (Brodmann areas 9 to 11) at various Braak senile plaque (SP) stages, using specific enzyme-linked immunosorbent assays. With advancing SP stages, the amounts of deposited $A \beta 1-43$ in the brain increased proportionally to those of A 1 1-42. Similarly, the amounts of deposited $A \beta 1-38$ correlated with those of $A \beta 1-40$. Surprisingly, the ratios of deposited $A \beta 1-38 / A \beta 1-42$ and $A \beta 1-40 / A \beta 1-43$ were proportional and discriminated the Braak SP stages accurately. This result indicates that the generation of $A \beta 1-38$ and $A \beta 1-40$ decreased and the generation of $A \beta 1-42$ and $A \beta 1-43$ increased with advancing SP stages. Thus, A $\beta$ s deposition might depend on $\gamma$-secretase activity, as it does in the cerebrospinal fluid. Here, the extracted $\gamma$-secretase from Alzheimer disease brains generates an amount of $A \beta 1-42$ and $A \beta 1-43$ compared with cognitively normal brains. This refractory $\gamma$-secretase localized in detergent-solubilized fractions from brain cortices. But activity modulated $\gamma$-secretase, which decreases $A \beta 1-42$ and $A \beta 1-43$ in the cerebrospinal fluid, localized in detergent-insoluble fractions. These drastic alterations reflect $A \beta$ situation in Alzheimer disease brains. (Am J Pathol 2020, 190: 1323-1331; https://doi.org/10.1016/j.ajpath.2020.02.009)
$\gamma$-Secretase is assumed to have two amyloid $\beta$-protein $(\mathrm{A} \beta)$ product lines that successively convert $A \beta 1-49$ and $A \beta 1-48$, which are generated by $\varepsilon$-cleavage from the carboxylterminal fragment of the $\beta$ amyloid protein precursor, generating conventional $\mathrm{A} \beta \mathrm{s}$ by trimming tripeptides or tetrapeptides in a stepwise manner. $A \beta 1-49$ is successively cleaved into mostly $A \beta 1-40$ via $A \beta 1-46$ and $A \beta 1-43$, whereas $A \beta 1-48$ is cleaved similarly into $A \beta 1-38$ via $A \beta 1-$ 45 and $A \beta 1-42 .{ }^{1,2}$ It is noteworthy that the most abundant species, $A \beta 1-40$, is derived not from $A \beta 1-42$, but from $\mathrm{A} \beta 1-43$. Moreover, $A \beta 1-38$ is derived largely from $A \beta 1-42$ and $A \beta 1-43$. $^{1-3}$ Herein, we measured these four $A \beta$ species (A $\beta 1-38, A \beta 1-40, A \beta 1-42$, and $A \beta 1-43)$, which are the major end products of stepwise $\beta$ amyloid protein precursor processing, in human brains at various senile plaque (SP) stages to assess SP stage-related alterations of $\gamma$-secretase activity and to obtain further insight into the mechanisms of parenchymal $A \beta$ deposition in the human brain.

\footnotetext{
This project, the Japanese Alzheimer's Disease Neuroimaging Initiative (J-ADNI), was supported in part by the New Energy and Industrial Technology Development Organization (NEDO); the Ministry of Education, Culture, Sports, Science, and Technology-Japan (MEXT)-Supported Program for the Strategic Research Foundation at Private Universities, 2012 to 2017 (Y.I.); the Strategic Research Program for Brain Sciences from the Japan Agency for Medical Research and Development (AMED); and AMED grant JP19dm017128 (N.K.).

Disclosures: None declared.
} 
Previously, we reported the unusual relationship between the four $A \beta$ s (A $\beta 1-38, A \beta 1-40, A \beta 1-42$, and $A \beta 1-43)$ in the cerebrospinal fluid $(\mathrm{CSF})^{4}$; the levels of $\mathrm{A} \beta 1-42$ and $A \beta 1-43$, and $A \beta 1-38$ and $A \beta 1-40$ are proportional in the CSF, which is consistent with a parallel stepwise processing via the two product lines of $\gamma$-secretase; and decreased levels of A $\beta 1-42$ and $A \beta 1-43$ in the CSF of patients affected by mild cognitive impairment (MCI) and Alzheimer disease (AD) may not be caused by selective deposition of $A \beta 1-42$ and $A \beta 1-43$ in pre-existing SPs, but may be caused by the modulation of $\gamma$-secretase activity, which enhances the conversion of $A \beta 1-42$ and $A \beta 1-43$ to $A \beta 1-38$ and $A \beta 1-40$, respectively. As a result, the levels of $A \beta 1-42$ and $A \beta 1-43$ in the CSF are reduced, whereas, reciprocally, the levels of $\mathrm{A} \beta 1-38$ and A $\beta 1-40$ are increased. ${ }^{4} \mathrm{We}$ assessed the $\gamma$-secretase activity in rafts isolated from autopsied cognitively normal and MCI/AD brains. The activity of raftassociated (detergent-insoluble) $\gamma$-secretase from MCI/AD brains to generate $A \beta 1-38$ and $A \beta 1-40$ from $A \beta 1-42$ and $\mathrm{A} \beta 1-43$, respectively, was significantly modulated compared with that from control brains. ${ }^{4}$ However, this observation raises a new question. Why do $\mathrm{A} \beta 1-42$ and A $\beta 1-43$ continue to accumulate in the brain (as assessed by amyloid positron emission tomography) despite significant modulation of $\gamma$-secretase activity ${ }^{5,6}$ It was particularly puzzling to observe that the modulation appears as early as the beginning of $\mathrm{A} \beta$ depositions in the cerebral parenchyma, which is decades before the development of clinical symptoms, ${ }^{7}$ and that, nevertheless, $\mathrm{A} \beta$ deposition continues to progress. To address this issue, we used the same approach as in the CSF study. ${ }^{4}$ On the basis of the levels of the four $\mathrm{A} \beta \mathrm{s}$ in the brain tissues, we sought to deduce the alteration in the activity of $\gamma$-secretase that is involved in A $\beta$ deposition in the interstitial fluid (ISF) compartment of the brain. We found that the amount of $A \beta 1-43$ deposited in the brain increased proportionally to that of $A \beta 1-42$ with advancing Braak SP stages. Similarly, the amount of A $\beta 1-38$ deposition increased proportionally to that of $A \beta 1-40$ with advancing SP stages. Surprisingly, the ratios of insoluble A $\beta 1-38 / A \beta 1-42$ levels versus $A \beta 1-40 / A \beta 1-43$ levels were proportional and discriminated the Braak SP stages accurately, as did the levels in the CSF. ${ }^{4}$ The plots representing $\mathrm{SP}$ stage $\mathrm{O}$ and $\mathrm{A}$ subjects were located distant from the origin on the regression line, whereas those representing SP stage $\mathrm{B}$ and $\mathrm{C}$ and $\mathrm{AD}$ subjects were close to the origin on the regression line or its surroundings. However, these plots were different from the patterns of the previous CSF study, in which the ratios of $A \beta 1-38 / A \beta 1-42$ versus $A \beta 1-40 / A \beta 1-$ 43 were plotted close to the origin for cognitively normal subjects and far from the origin for $\mathrm{MCI} / \mathrm{AD}$ patients. ${ }^{4}$ Consequently, in this study, amyloid-free (SP stage O) subjects and less subjects (SP stage A) plotted far from the origin, and amyloid-bearing subjects (SP stages B, C, and $\mathrm{AD})$ plotted close to the origin. These ratios suggest that ISF compartment-associated $\gamma$-secretase activity is altered for active $A \beta 1-42$ and $A \beta 1-43$ generation after the subjects of amyloid-bearing SP stage B. Herein, the in vitro $\gamma$-secretase activity assay allowed that ISF compartment-associated (detergent-soluble raft-nonassociated) $\gamma$-secretase from the brain cortices generates large amounts of $A \beta 1-42$ and $\mathrm{A} \beta 1-43$ in $\mathrm{AD}$ brains compared with cognitively normal control (SP stage O) brains; thus, the two $\gamma$-secretases, raftassociated and raft-nonassociated $\gamma$-secretase, generate $A \beta s$ in the brain. Both $\gamma$-secretase activities have been altered to modulation and refractory modulation each other in the amyloid-bearing brain.

\section{Materials and Methods}

\section{Braak SP Stages and Tissue Preparation}

In the present study, the extent of $A \beta$ deposition detected by an anti-A $\beta$ monoclonal antibody $(12 \mathrm{~B} 2 ; 1: 50$ dilution; IBL, Gunma, Japan) was defined by the Braak SP (amyloid) stages. ${ }^{8,9}$ At SP stage O, there were almost no SPs throughout the isocortex. At stage $A$, a low density of $A \beta$ deposits was found in the isocortex, particularly in the basal portions of the frontal, temporal, and occipital lobes. In addition, some SPs were found in the presubiculum and pre$\beta$ and pre- $\gamma$ layers of the entorhinal complex. Stage B showed an increase in $\mathrm{A} \beta$ deposits in almost all isocorticalassociated areas, and only the primary sensory areas and the primary motor field remained almost devoid of deposits. Some deposits were found in the hippocampal formation, and $A \beta$ deposits may be found in the entorhinal cortex. At stage $\mathrm{C}$, virtually all isocortical areas were affected, whereas deposits in the hippocampal formation showed the same pattern as that of stage B. AD brains were invariably at stage $\mathrm{C}$.

Human cortical specimens were obtained from the prefrontal cortex (Brodmann areas 9 to 11) within 12 hours postmortem (patients were placed in a room at $4^{\circ} \mathrm{C}$ within 2 hours after death) and stored at $-80^{\circ} \mathrm{C}$ until use. Cortical pieces, approximately $200 \mathrm{mg}$ each, were sampled from the following frozen brains at the Brain Bank of the Tokyo Metropolitan Institute of Gerontology: Braak SP stage O [Braak neurofibrillary tangle $(\mathrm{NFT})$ stage $<$ I, Clinical Dementia Rating $(\mathrm{CDR})=0,68$ to 94 years old, $n=10$ ], SP stage A (Braak NFT stage $<$ I, CDR $<0.5,71$ to 84 years old, $n=10$ ), SP stage B (Braak NFT stage $<$ III, CDR $<0.5,66$ to 89 years old, $n=10$ ), SP stage C (Braak NFT stage $<\mathrm{IV}, \mathrm{CDR}>0.5,70$ to 91 years old, $n=10$ ), and AD (Braak NFT stage $>$ IV, CDR $>1,75$ to 87 years old, $n=10) .{ }^{10}$ The attached leptomeninges and small blood vessels were carefully dissected. Each sample was homogenized with a motor-driven Teflon/glass homogenizer in four volumes of Tris-saline (TS) [TS: $50 \mathrm{mmol} / \mathrm{L}$ Tris- $\mathrm{HCl}$ $(\mathrm{pH} 7.6)$ and $0.15 \mathrm{~mol} / \mathrm{L} \mathrm{NaCl}$ containing a cocktail of protease inhibitors. Each homogenate was then centrifuged at $540,000 \times g$ for 20 minutes in a TLX centrifuge (Beckman, Brea, CA). The resultant supernatant was saved as the TS-soluble fraction and subjected to an enzyme- 
A

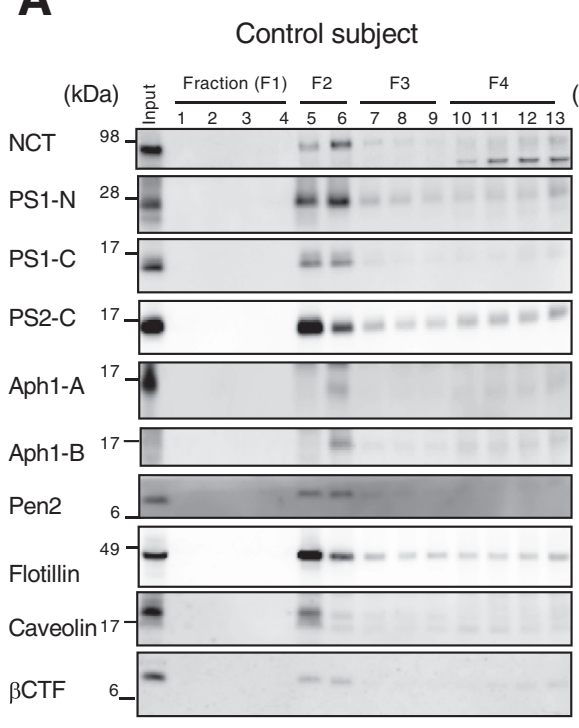

AD patient

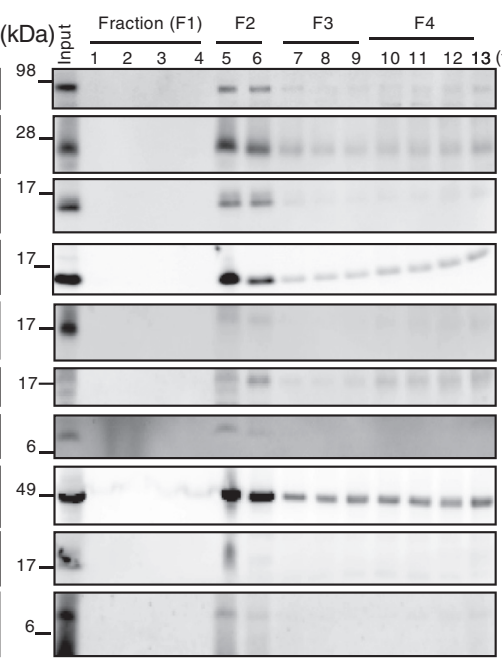

B

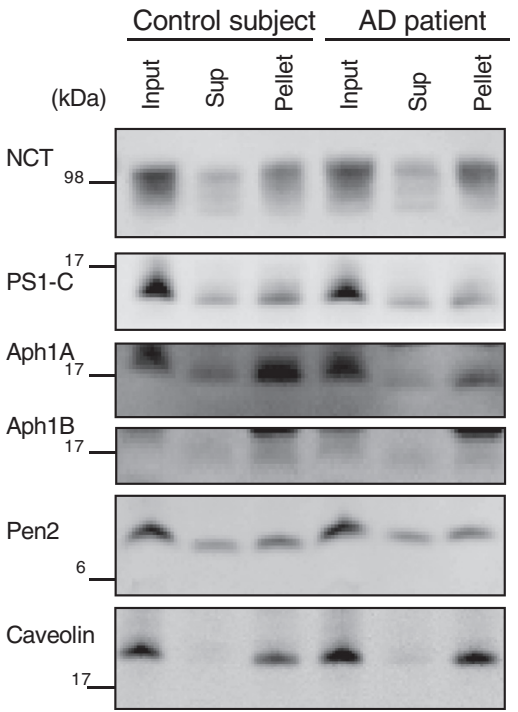

Figure 1 Distinct raft-nonassociated $\gamma$-secretase complex fractionated by sucrose density gradient centrifugation and the CHAPSO-solubilizing method. A: After sucrose density gradient centrifugation, each fraction (F) was collected from the top and subjected to 12\% SDS-PAGE. F2 contained raft-associated $\gamma$-secretase (CHAPSO-resistant) components, whereas F4 contained raft-nonassociated $\gamma$-secretase (CHAPSO-soluble) components. B: Human brain microsomal fractions were solubilized with $1 \%$ CHAPS0. After centrifugation, supernatants were collected (raft-nonassociated $\gamma$-secretase). The fractionated $\gamma$-secretase contained nicastrin (NCT), presenilin 1/2 (PS1/2), pen-2, and Aph1A/B. AD, Alzheimer disease; $\beta C T F, ~ \beta$ amyloid protein precursor; Sup, supernatant.

linked immunosorbent assay (ELISA) to qualify TS-soluble $\mathrm{A} \beta 1-38, \mathrm{~A} \beta 1-40, \mathrm{~A} \beta 1-42$, and $\mathrm{A} \beta 1-43$, as described previously. ${ }^{4}$ The resulting pellet was washed with TS and extracted with $6 \mathrm{~mol} / \mathrm{L}$ guanidine- $\mathrm{HCl}$ in $50 \mathrm{mmol} / \mathrm{L}$ Tris$\mathrm{HCl}(\mathrm{pH}$ 7.6). The homogenate was centrifuged at $540,000 \times g$ for 20 minutes. The supernatant was diluted to $0.5 \mathrm{~mol} / \mathrm{L}$ in guanidine-HCl. An ELISA for the four TS-insoluble $A \beta$ species, $A \beta 1-38, A \beta 1-40, A \beta 1-42$, and A $\beta 1-43$, was performed.

The human cortical specimens used for the quantification of raft-nonassociated $\gamma$-secretase activity were obtained from those brains that were removed, processed, and placed in a container at $-80^{\circ} \mathrm{C}$ within 12 hours postmortem (patients were placed in a room at $4^{\circ} \mathrm{C}$ within 2 hours after death) at the Brain Bank of the Tokyo Metropolitan Institute of Gerontology. Written informed consent from the patient or the patient's family was obtained before the present study, which was approved by the ethics committees of the Doshisha University and Tokyo Metropolitan Institute of Gerontology.

\section{Immunohistochemistry}

Small blocks of frontal and temporal cortices from five AD and five non-AD (aged) subjects were fixed with $4 \%$ formaldehyde in a buffer for 2 days and embedded in paraffin. After pretreatment with $100 \%$ formic acid for 5 minutes to enhance immunolabeling, dewaxed serial tissue sections $(4 \mu \mathrm{m}$ thick) were incubated with monoclonal antibodies specific for $\mathrm{A} \beta 38,{ }^{4} \mathrm{~A} \beta 40$ (M40), ${ }^{11}$ and $\mathrm{A} \beta 42$ $(\mathrm{M} 42),{ }^{11}$ and a polyclonal antibody specific for $\mathrm{A} \beta 43{ }^{4,7}$ M42 did not cross-react with $A \beta 43$, and the $A \beta 43$ polyclonal antibody did not cross-react with $A \beta 42$ on the blot. ${ }^{4}$
The antibodies were visualized using the $\mathrm{ABC}$ Elite kit (Vector Laboratories, Burlingame, CA) using 3, 3'-diaminobenzidine as a chromogen.

\section{Isolation of $\gamma$-Secretase Complexes Using Sucrose Density Gradient Centrifugation}

The sucrose density gradient method was described previously. ${ }^{4}$ Briefly, after careful removal of the leptomeninges and blood vessels, small $(<0.5 \mathrm{~g})$ blocks from the prefrontal cortices (Brodmann areas 9 to 11) were homogenized in approximately 10 volumes of $10 \%$ sucrose in 2-morpholinoethanesulfonic acid (MES)-buffered saline $(25 \mathrm{mmol} / \mathrm{L} \mathrm{MES,} \mathrm{pH} 6.5$, and $0.15 \mathrm{~mol} / \mathrm{L} \mathrm{NaCl})$ containing 1\% 3-[(3-cholamidopropyl) dimethylammonio]-2-hydroxypropanesulfonate (CHAPSO) and various protease inhibitors. The homogenate was adjusted to $40 \%$ sucrose by the addition of an equal volume of $70 \%$ sucrose in MES-buffered saline, placed at the bottom of an ultracentrifuge tube, and overlaid with $4 \mathrm{~mL}$ of $35 \%$ sucrose and $4 \mathrm{~mL}$ of $5 \%$ sucrose in MES-buffered saline. The discontinuous gradient was centrifuged at $260,800 \times g$ for 20 hours at $4^{\circ} \mathrm{C}$ with an SW $41 \mathrm{Ti}$ rotor (Beckman). After ultracentrifugation, each $1-\mathrm{mL}$ fraction was recovered from the top of ultracentrifuge tube to the bottom (Figure 1A).

\section{Isolation and Quantification of Raft-Nonassociated $\gamma$-Secretase Activity}

Microsomal fractions from cortical pieces were obtained, as described previously, ${ }^{12}$ with modifications. Briefly, small 
A

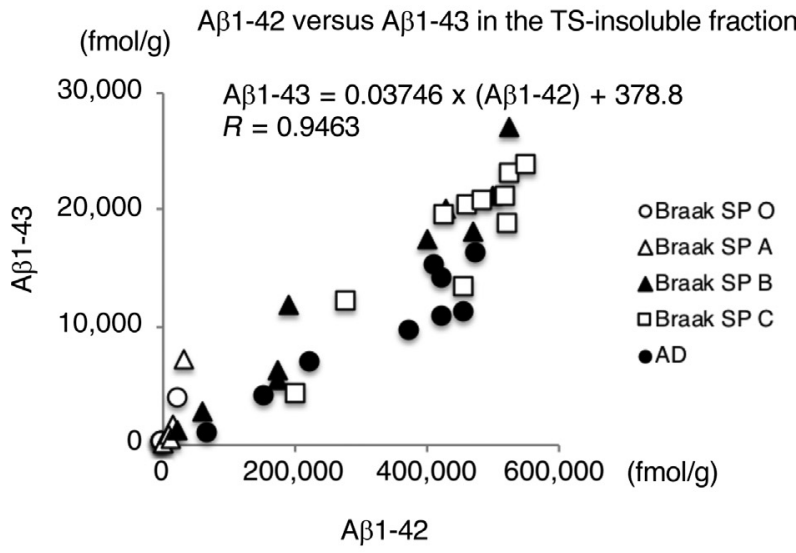

B

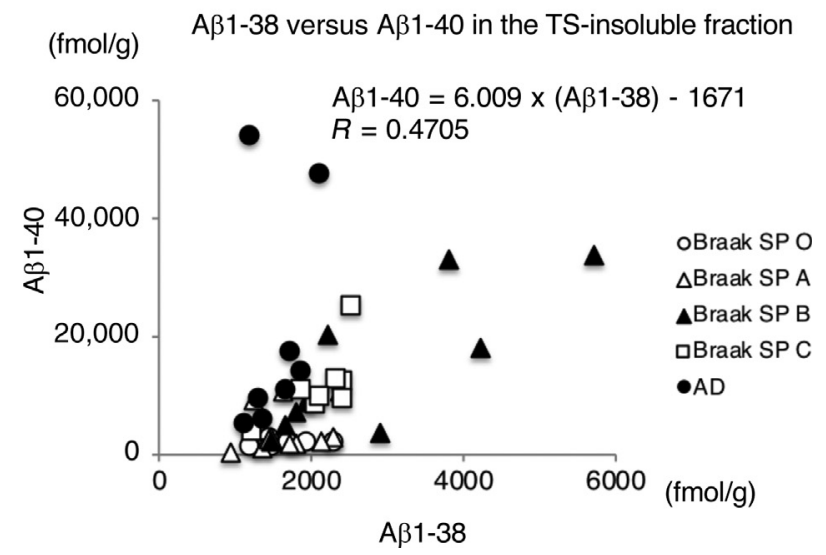

Figure 2 Relationships between the levels of $A \beta 1-42$ and $A \beta 1-43$ (A) and $A \beta 1-38$ and $A \beta 1-40$ (B) in the Tris-saline (TS) -insoluble fractions from Braak senile plaque (SP) stages $0, A, B$, and $C$, and Alzheimer disease (AD) brains. All fractions were appropriately diluted, and $A \beta 1-38, A \beta 1-40$, $A \beta 1-42$, or $A \beta 1-43$ was measured using enzyme-linked immunosorbent assay. The levels of $A \beta s$ in the TS-insoluble fractions were correlated with the SP stages.

pieces of the cortices were homogenized in buffer A [20 $\mathrm{mmol} / \mathrm{L}$ piperazine-1, 4-bis(2-ethanesulfonic acid) (PIPES), $\mathrm{pH} 7.0,0.14 \mathrm{~mol} / \mathrm{L} \mathrm{KCl}, 0.25 \mathrm{~mol} / \mathrm{L}(8.56 \%)$ sucrose, and 5 $\mathrm{mmol} / \mathrm{L}$ EGTA] using a Teflon/glass homogenizer. The homogenates were centrifuged at $800 \times g$ for 10 minutes. The postnuclear supernatants were centrifuged at $100,000 \times$ $g$ for 1 hour. The resulting pellets, which represented the microsomal fractions, were suspended in buffer $(20 \mathrm{mmol} / \mathrm{L}$ PIPES, pH 7.0, $0.25 \mathrm{~mol} / \mathrm{L}$ sucrose, and $1 \mathrm{mmol} / \mathrm{L}$ EGTA). Their protein concentrations were adjusted to $10 \mathrm{mg} / \mathrm{mL}$. The membranes were solubilized by the addition of an equal volume of $2 \times$ Nobuto Kakuda (NK) buffer $(20 \mathrm{mmol} / \mathrm{L}$ PIPES, pH 7.0, $0.25 \mathrm{~mol} / \mathrm{L}$ sucrose, $1 \mathrm{mmol} / \mathrm{L}$ EGTA, $2 \%$ CHAPSO, and protease inhibitors) and incubated on ice for 1 hour. After centrifugation at $100,000 \times g$ for 1 hour, the supernatants were saved (1\% CHAPSO lysate). Each $1 \%$ CHAPSO-solubilized fraction, adjusted to $0.25 \%$ CHAPSO, was incubated with $1 \mu \mathrm{mol} / \mathrm{L}$ C99FLAG for 1 hour at $37^{\circ} \mathrm{C}$ in the presence or absence of $10 \mu \mathrm{mol} / \mathrm{L}$ GSM- 1 . The produced $A \beta$ s were separated using SDS-PAGE and subjected to quantitative Western blot analysis using the following antibodies: 3B1 for $\mathrm{A} \beta 38$, BA27 for $\mathrm{A} \beta 40,44 \mathrm{~A} 3$ for $\mathrm{A} \beta 42$, anti-A $\beta 43$ polyclonal antibody for $A \beta 43,{ }^{4} 9 \mathrm{C} 3$ for nicastrin, ${ }^{13}$ monoclonal antibodies 1563 and 5232 for presenilin 1 (Millipore, Burlington, MA), D30G3 for presenilin 2 (Cell Signaling, Danvers, MA), B126 for pen-2 and B80 for Aph1A, ${ }^{13,14}$ O2E2 for Aph1B (Covance, Princeton, NJ), 82E1 for $\beta$ amyloid protein precursor (IBL, Gunma, Japan), caveolin-1 for caveolin (Santa Cruz Biotechnology, Dallas, TX), and flotillin-1 for flotillin (BD, Franklin Lakes, NJ).

Quantification of GSM-1-Induced Shifts for the Ratios of $A \beta 38 / A \beta 42$

A previously reported procedure was used to quantify GSM1 efficacy. ${ }^{7}$ The ratios of $A \beta 1-38 / A \beta 1-42$ in the presence of GSM-1 minus in the absence of GSM-1 were calculated.

\section{Statistical Analysis}

All statistical analyses were performed using SPSS software version 20 (SPSS, Chicago, IL) and GraphPad Prism 8 (GraphPad, San Diego, CA). An analysis of variance with a Kruskal-Wallis test was used to assess the equality of mean values of continuous variables among five groups (ie, SP stage O, SP stage A, SP stage B, SP stage C, and AD). A Dunn's multiple comparisons test for TS-soluble and TS-insoluble $A \beta$ amounts showed a significant difference between SP stage O, SP stage A, SP stage B, SP stage $\mathrm{C}$, and $\mathrm{AD}(P<0.005)$. A $U$-test for raftnonassociated $\gamma$-secretase activity showed a significant difference between normal controls and $\operatorname{AD}(P<0.05)$. A paired $t$-test was used to examine raft-associated $\gamma$-secretase activity, which was recovered from the CHAPSO insoluble pellet fraction, and the effect of GSM-1 $(P<0.05)$.

\section{Results}

The Levels of Insoluble $A \beta 1-42$ and $A \beta 1-43$ Are Proportional

SPs in the brain cortices probably consist of A $\beta 1-42 .{ }^{15}$ To assess deposited $A \beta s$ in the cerebral cortex, $A \beta$ s were extracted from autopsied frozen brain cortices (SP stages $\mathrm{O}$, $\mathrm{A}, \mathrm{B}$, and $\mathrm{C}$, and $\mathrm{AD}$ ) with guanidine hydrochloride after soluble $A \beta s$ were discarded. $A \beta s$ in these extracts were measured using specific ELISA. ${ }^{4}$ The concentration of guanidine-soluble $A \beta 1-42$ (termed as TS-insoluble $A \beta 1-42$ ) was exactly proportional to that of TS-insoluble A $\beta 1-43$, and the ratio of $A \beta 1-42 / A \beta 1-43$ was approximately $20: 1$ (Figure $2 \mathrm{~A})(\mathrm{A} \beta 1-43=0.03746 \times \mathrm{A} \beta 1-42+378.8$; $R=0.9463)$. The concentration of TS-insoluble A $\beta 1-42$ and $A \beta 1-43$ significantly differed between advanced SP 


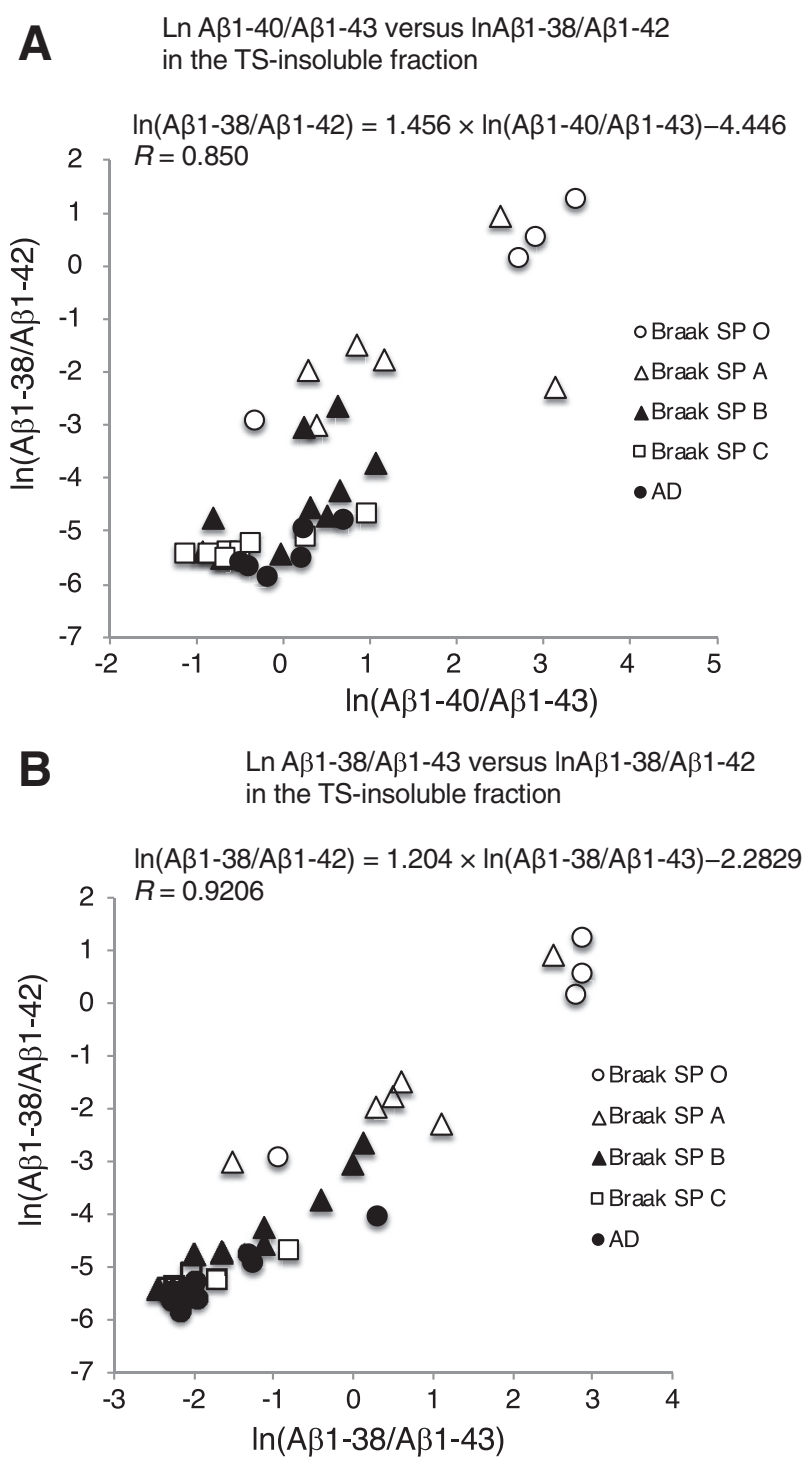

Figure 3 Relationships between the ratios of product $A \beta /$ precursor $A \beta$ sets in the Tris-saline (TS)-insoluble fractions. A: Braak senile plaque (SP) stages were distinguishable precisely by the ratios of $\ln (A \beta 1-38 / A \beta 1-42)$ and $\ln (A \beta 1-40 / A \beta 1-43)$ in the TS-insoluble fractions. B: The ratios of $\ln (A \beta 1-38 / A \beta 1-42)$ and $\ln (A \beta 1-38 / A \beta 1-43)$ in the TS-insoluble fractions. $A D$, Alzheimer disease.

stages (Supplemental Tables S1 and S2). In this TS-insoluble A $\beta 1-42$ ELISA measurement, all of SP stage $\mathrm{O}$ and nine of SP stage A subjects had levels of A $\beta 1-42$ that were below the limit of detection (data not shown).

On the other hand, the concentration of TS-insoluble $A \beta 1-38$ and $A \beta 1-40$ also correlated, except for some $A D$ brains. The ratio of $A \beta 1-38 / A \beta 1-40$ was approximately 1:6 (Figure $2 \mathrm{~B})(\mathrm{A} \beta 1-40=6.009 \times \mathrm{A} \beta 1-38-1671$; $R=0.4705$ ). The level of TS-insoluble $\mathrm{A} \beta 1-38$ and A $\beta 1-40$ significantly differed between advanced SP stages, with the exception of $\mathrm{A} \beta 1-38$ for some $\mathrm{AD}$ brains (Supplemental Tables S1 and S2). It is possible that the relatively weak correlation between $\mathrm{A} \beta 38$ and $\mathrm{A} \beta 40$ is caused by their preferential accumulation in leptomeningeal blood vessels and lower insolubilities of their aggregates in the cerebral parenchyma (Supplemental Figure S1). In contrast, $A \beta 42$ and $A \beta 43$ are preferentially deposited in the cerebral parenchyma rather than in the vessels (Supplemental Figure S1). These observed proportionalities between TS-insoluble $A \beta 1-42$ and $A \beta 1-43$ reminded us of the relationships between the four $A \beta s$ in the CSF, as reported previously, ${ }^{4}$ because these proportionalities are characteristic of the parallel stepwise processing of $\beta$ amyloid protein precursor by $\gamma$-secretase. ${ }^{4}$ Thus, Figure $2 \mathrm{~A}$ may indicate that as SP stages advance, $\gamma$-secretase activity to generate $A \beta 1-38$ and $A \beta 1-40$ from $A \beta 1-42$ and $A \beta 1-43$ decelerates, and, therefore presumably enhances $A \beta 1-42$ and A $\beta 1-43$ accumulation in the cerebral parenchyma.

\section{Insoluble $A \beta 1-38 / A \beta 1-42$ versus $A \beta 1-40 / A \beta 1-43$ Accurately Discriminates Various SP Stages}

To clarify whether not only $A \beta s$ in CSF or also $A \beta$ deposits in the brain depend on $\gamma$-secretase activity, we plotted the two ratios of the product and precursor set for $A \beta 1-38 / A \beta 1$ 42 versus $A \beta 1-40 / A \beta 1-43$ of the TS-insoluble fractions (Supplemental Figure S2). However, because of the large amount of $A \beta 1-42$ and $A \beta 1-43$ deposits in subjects with Braak SP stages $\mathrm{B}$ and $\mathrm{C}$ and $\mathrm{AD}$, these subjects were plotted close to the origin without discrimination. To assess further the distribution for these plots, the values of TS-insoluble $A \beta s$ were plotted after being logarithmically transformed $[\ln (A \beta 1-38 / A \beta 1-42)$ versus $\ln (A \beta 1-40 / A \beta 1$ 43)] (Figure $3 \mathrm{~A})$. In these plots, an unusual proportion was allowed in Figure $3 \mathrm{~A}[\ln (\mathrm{A} \beta 1-38 / \mathrm{A} \beta 1-42)=1.465$ $\times \ln (\mathrm{A} \beta 1-40 / \mathrm{A} \beta 1-43)-4.446 ; R=0.850$ ], similar to plots of $\mathrm{A} \beta \mathrm{s}$ in the CSF. ${ }^{4}$ All subjects were on the regression line or its surroundings. In detail, AD- and SP-abundant subjects (SP stages B and C) were plotted close to the origin on the regression line and its surroundings, whereas SP-free (SP stage O) and SP stage A subjects were plotted far from the origin on the regression line and its surroundings (Figure 3A and Supplemental Table S3). Thus, most interestingly, the plot images showed opposite results from those obtained for A $\beta 1-38 / A \beta 1-42$ versus $A \beta 1-40 / A \beta 1-43$ in the CSF from normal controls and MCI/AD subjects. ${ }^{4}$ In our previous CSF measurement study, MCI/AD subjects were plotted far from the origin and normal controls were plotted close to the origin on the regression line for $A \beta 1-38 / A \beta 1-42$ versus $\mathrm{A} \beta 1-40 / \mathrm{A} \beta 1-43{ }^{4}{ }^{4}$ Thus, the plots of CSF and TS-insoluble $A \beta s$ are different. In the cerebral parenchyma, $A \beta s$ form SPs decades before the onset of AD. Previous studies have claimed that $A \beta \mathrm{s}^{\prime}$ accumulation and deposition are caused by an imbalance of $A \beta s$ ' production and clearance associated with aging. ${ }^{15,16}$ However, in addition to aging, this plot implies that $\gamma$-secretase activity also causes $A \beta s$ ' accumulation and deposition in the brain. In addition, $\gamma$-secretase generated $A \beta 1-38$ not only from $A \beta 1-42$ but also from $\mathrm{A} \beta 1-43 .{ }^{2,3}$ Therefore, this product and precursor set of TS-insoluble $A \beta s$ also assessed the relationship for 


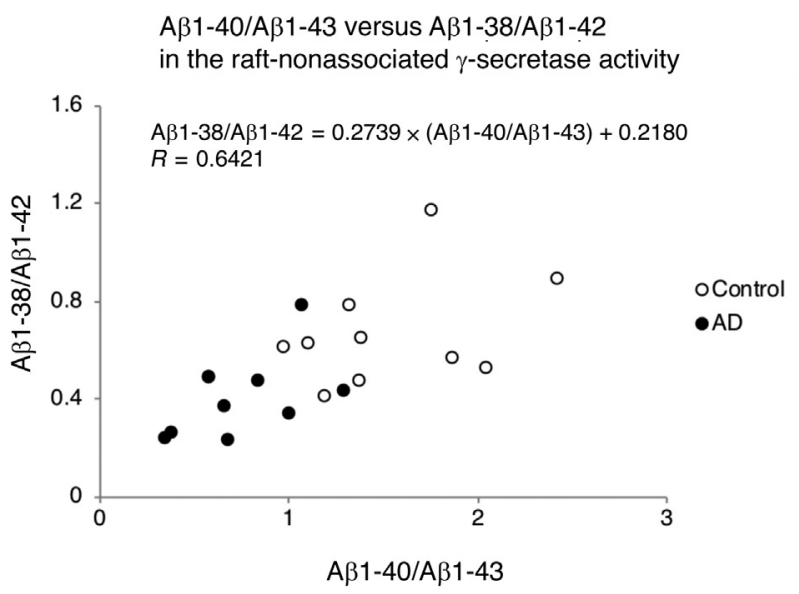

$P=0.0002$ for $A \beta 1-40 / A \beta 1-43$ $P=0.0052$ for $A \beta 1-38 / A \beta 1-42$

Figure $4 \quad A \beta 1-38 / A \beta 1-42$ versus $A \beta 1-40 / A \beta 1-43$ plot based on the direct quantification of raft-nonassociated $\gamma$-secretase activity. The raftnonassociated $\gamma$-secretase prepared from the microsomal fractions of control (Braak senile plaque stage 0 ) and Alzheimer disease (AD) brains was incubated with $\beta$ amyloid protein precursor for 1 hour at $37^{\circ} \mathrm{C}$. The produced $A \beta$ s were quantified using Western blot analysis with specific antibodies. The plots clearly discriminated between control and AD brains. $A D$ plots (closed circles) are located near the origin, whereas control plots (open circles) are located distant from the origin. The original values for $\mathrm{A} \beta \mathrm{s}$ are in the pmol/L range. $n=10 \mathrm{AD}$ and control plots.

TS-insoluble $A \beta$ s. As expected, the ratio of $A \beta 1-38 / A \beta 1-42$ versus $A \beta 1-38 / A \beta 1-43$ also allowed a correlation pattern as advanced $\mathrm{SP}$ stage $[\ln (\mathrm{A} \beta 1-38 / \mathrm{A} \beta 1-42)=1.204 \times$ $\ln (\mathrm{A} \beta 1-38 / \mathrm{A} \beta 1-43)$ - 2.2829] (Figure 3B). Thus, $\mathrm{A} \beta$ deposition in the cerebral parenchyma also possibly depends on $\gamma$-secretase activity. More important, these $A \beta s$ generated by $\gamma$-secretase differ from $A \beta s$ generated by raft-associated $\gamma$-secretase, which is reflected by the $A \beta s$ found in the CSF. Raft-associated $\gamma$-secretase has been modulated to actively generate $A \beta 1-38$ and $A \beta 1-40$ from $\mathrm{A} \beta 1-42$ and $\mathrm{A} \beta 1-43$, respectively, in $\mathrm{MCI} / \mathrm{AD}$ brains; and it decreased the levels of $A \beta 1-42$ and $A \beta 1-43$ in the CSF. ${ }^{4}$ However, another subset of $\gamma$-secretase, which is reflected by the $A \beta$ deposits in the cerebral parenchyma, suggests that the $\gamma$-secretase cleavage efficacy decreases and with it the generation of $A \beta 1-38$ and $A \beta 1-40$ from $A \beta 1-42$ and $A \beta 1-43$, respectively, by resistance to modulation, rather than by nonmodulation. This $\gamma$-secretase in the ISF compartment continues generating $A \beta 1-42$ and $A \beta 1-43$ to a greater extent and secretes $A \beta s$ in the ISF compartment, which may continue to accumulate in the brain. Thus, it is possible that there are two different types of $\gamma$-secretase in the brain. One is raft-associated $\gamma$-secretase, which secretes A $\beta$ s into the CSF. Another is $\gamma$-secretase, which undergoes resistance to modulation in amyloid-bearing brains and generates longer $A \beta$ species and secretes them into the ISF compartment. These two distinct $\gamma$-secretases' functions may be regulated in opposite directions as SP stages advance.

\section{Raft-Nonassociated $\gamma$-Secretase Is Refractory} Modulated in Amyloid-Bearing Brains

To clarify whether there is another subset of the $\gamma$-secretase, brain cortices were fractionated into raft-associated and CHAPSO-solubilized $\gamma$-secretase by sucrose density gradient centrifugation. ${ }^{4}$ Most of the $\gamma$-secretase fraction, consisting of four components (presenilin 1/2, nicastrin, Aph1A/B, and pen-2), was recovered in the CHAPSO-insolubilized raft fraction (Figure 1A). Previously, we reported that these raftassociated $\gamma$-secretase activities have been modulated in $\mathrm{MCI} / \mathrm{AD}$ brains to advance the generation of $\mathrm{A} \beta 1-38$ and $A \beta 1-40$ from $A \beta 1-42$ and $A \beta 1-43$, respectively, compared with cognitively normal control brains and possibly reflected to the CSF A $\beta s .{ }^{4}$ However, low levels of another $\gamma$-secretase were also recovered in the CHAPSO-solubilized fraction (Figure 1A).

We measured this CHAPSO-solubilized $\gamma$-secretase activity to validate our hypothesis on the distinct modulation of the $\gamma$-secretase activity. First, we could not measure it directly because the concentration and activity of CHAPSO-solubilized $\gamma$-secretase was much lower after sucrose density gradient centrifugation for 20 hours. Therefore, we changed the isolation protocol: highconcentration raft-nonassociated $\gamma$-secretase in the CHAPSO-soluble fraction was recovered within 2 hours from the membrane fractions of brain cortices solubilized with CHAPSO, as reported previously (CHAPSO-solubilizing method). ${ }^{12}$ This CHAPSO-solubilized $\gamma$-secretase is referred to as raft-nonassociated $\gamma$-secretase. Raft-nonassociated $\gamma$-secretase was recovered in the supernatant as the solubilized $\gamma$-secretase, but raft-associated $\gamma$-secretase was pelleted in this method because it cannot be solubilized with CHAPSO (Figure 1B).

This CHAPSO-solubilized, raft-nonassociated $\gamma$-secretase activity was measured in normal controls (SP stage O) and $\mathrm{AD}$ brains using an in vitro cell-free assay system that was established previously. ${ }^{12}$ The ratios of generated $A \beta 1$ 38/A $\beta 1-42$ and $A \beta 1-40 / A \beta 1-43$ were quantified via immunoblotting and plotted (Figure 4). As expected, all plots were distributed as a whole in a diagonal manner. The plots representing $\mathrm{AD}$ subjects were located near the origin, whereas the plots representing normal controls were far from the origin in Figure $4(P=0.0002$ for $\mathrm{A} \beta 1-40 / \mathrm{A} \beta 1$ $43 ; P=0.0052$ for A $\beta 1-38 / \mathrm{A} \beta 1-42)$, as observed for the plots of the ELISA results of TS-insoluble fraction (Figure 3A). These results allowed both from $A \beta 1-43$ to $A \beta 1-40$ and from $A \beta 1-42$ to $A \beta 1-38$ cleavage efficacy significantly decreased in $\mathrm{AD}$ raft-nonassociated $\gamma$-secretase, suggesting that it actively produces $A \beta 1-42$ and $A \beta 1$ 43 in AD. The image shown in Figure 4 is the opposite pattern plot of raft-associated $\gamma$-secretase in vitro assay. ${ }^{4}$ It would be more appropriate to call this species refractorymodulated $\gamma$-secretase. To assess the remaining raftassociated $\gamma$-secretase activity, it was recovered from once raft-nonassociated $\gamma$-secretase removed pellet fractions and 


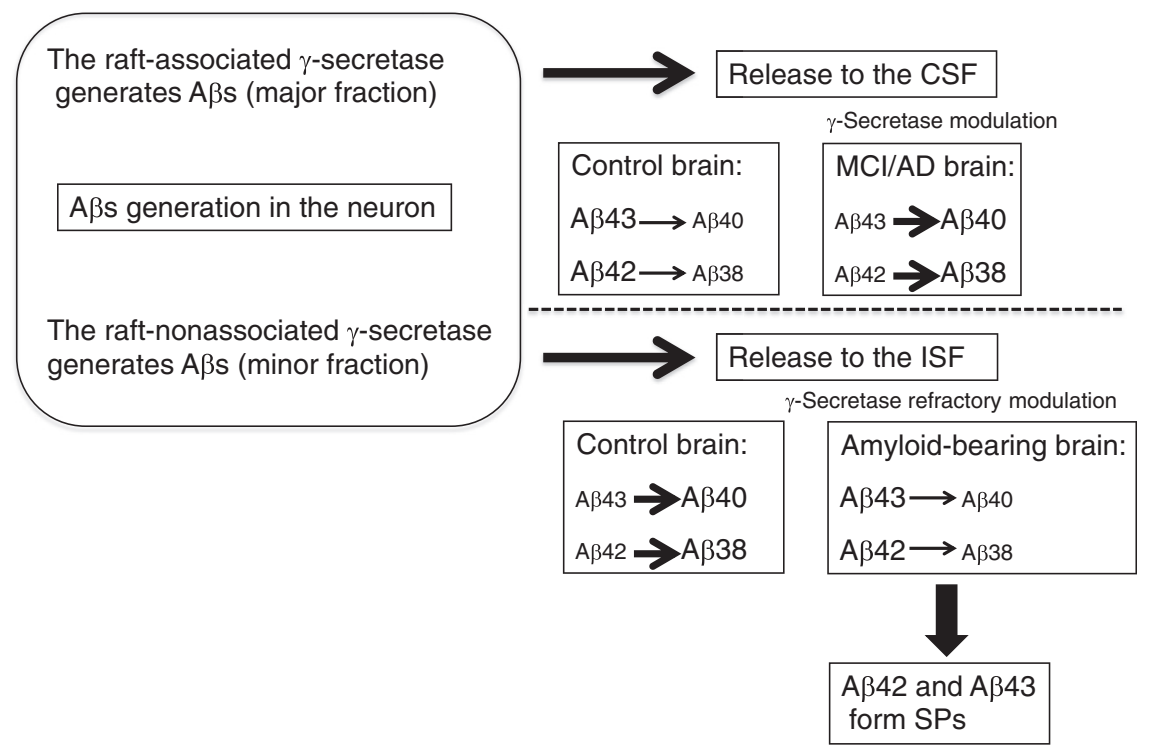

Figure 5 Scheme of generated $A \beta s$ releasing into cerebrospinal fluid (CSF) and interstitial fluid (ISF). In the neurons, $A \beta S$ generated by raftassociated $\gamma$-secretase are released into the CSF via still unknown factors. $A \beta$ s generated by raftnonassociated $\gamma$-secretase are released into the extracellular space and the ISF. A $A 1-38$ and $A \beta 1-$ 40 tend to accumulate in the vessel walls, and $A \beta 1-42$ and $A \beta 1-43$ are deposited preferably in the cerebellum parenchyma, which forms senile plaques (SPs). AD, Alzheimer disease; MCI, mild cognitive impairment. measured these raft-associated $\gamma$-secretase activities with in vitro assay. As expected, the raft-associated $\gamma$-secretase was modulated in $\mathrm{AD}$ brains compared with normal controls, as in our previous study $(P=0.0232$ for $\mathrm{A} \beta 1-40 /$ A $\beta 1-43 ; P<0.0001$ for A $\beta 1-38 / \mathrm{A} \beta 1-42$ ) (Supplemental Figure S3). ${ }^{4}$ Thus, two distinct types of $\gamma$-secretase, raftassociated and raft-nonassociated $\gamma$-secretase, are active in the brain, but their activities have been altered depending on the SP stages.

GSM-1, a strong $\gamma$-secretase modulator, has previously been shown to limit $A \beta 42$-decreasing and $A \beta 38$-increasing modulation efficacy toward $\mathrm{AD}$, because it had already been modulated to generate shorter A $\beta \mathrm{s}^{7}$ It was hypothesized that, because of the raft-nonassociated $\gamma$-secretase activity described above, GSM-1 would cause a larger modulation of AD raft-nonassociated $\gamma$-secretase because its activity has been resisted. In normal control subjects, GSM-1 definitely allowed a large modulation shift from $A \beta 1-42$ to $A \beta 1-38$ (A $\beta 1-38 / A \beta 1-42$ with dimethyl sulfoxide versus $A \beta 1-38 /$ A $\beta 1-42$ with GSM-1; $P<0.0001$, paired $t$-test) (Supplemental Figure S4). However, raft-nonassociated $\gamma$-secretase in $\mathrm{AD}$ brains, which had been refractory modulated to generate longer $A \beta$ s, showed only a poor modulation shift value (A $\beta 1-38 / A \beta 1-42$ with dimethyl sulfoxide versus A $\beta 1-38 / \mathrm{A} \beta 1-42$ with GSM-1; $P<0.0001$, paired $t$-test) (Supplemental Figure S4). Moreover, the shift value in $\mathrm{AD}$ brains was smaller than that in control brains (control versus $\mathrm{AD}, P=0.0013$, paired $t$-test). Thus, GSM-1 is unlikely to affect the modulation efficacy toward AD brain raft-nonassociated $\gamma$-secretase (ie, likely involved in the parenchymal $A \beta$ deposition).

\section{Discussion}

This manuscript demonstrates the following: i) the levels of deposited $A \beta 1-42$ and $A \beta 1-43$ in the brains of cognitively normal subjects and $\mathrm{AD}$ patients were proportional in the TS-insoluble fractions; ii) the levels of accumulated A $\beta 1-38$ and A $\beta 1-40$ correlated in the TS-insoluble fractions; iii) the plots of $A \beta 1-38 / A \beta 1-42$ versus $A \beta 1-40 / A \beta 1-43$ for the TS-insoluble fractions discriminated the various SP stages accurately: stage $\mathrm{B}$ and $\mathrm{C}$ and $\mathrm{AD}$ subjects were located close to the origin on the regression line, whereas SP stage $\mathrm{O}$ and $\mathrm{A}$ subjects were located distant from the origin on the regression line; iv) in sharp contrast to the plots of $A \beta 1-38$ / $A \beta 1-42$ versus $A \beta 1-40 / A \beta 1-43$ for raft-associated $\gamma$-secretase, ${ }^{4}$ the plots for raft-nonassociated $\gamma$-secretase activity measured in vitro showed that $\mathrm{AD}$ subjects were located close to the origin on the regression line, whereas cognitively normal subjects were located far from the origin on the line. Thus, raft-nonassociated $\gamma$-secretase is modulated to refractory alteration. Finally, v) GSM-1 showed poor modulation efficacy toward raft-nonassociated $\gamma$-secretase in AD brains, because this activity has been refractory modulated against the raft-associated $\gamma$-secretase modulation.

Compared with the previous CSF study, ${ }^{4}$ an apparent contradiction seems to exist: if the levels of intracellular soluble $A \beta$ s are negligible, the levels of $A \beta 1-42$ and $A \beta 1$ 43 in the TS-soluble fractions are not significantly decreased, as predicted by the profound modulation of raftassociated $\gamma$-secretase in SP-bearing brains (Supplemental Figure S5). A reasonable explanation for this is that the CSF and ISF compartments do not communicate freely, although leaky pathways seem to be present. ${ }^{17}$ The ISF compartment drains through the perivascular drainage pathways to the lymphatic flow on the meningeal blood vessel walls and to the regional lymph nodes in the neck. ${ }^{18,19}$ The Virchow-Robin space is completely separated from the CSF compartment by the pia mater. ${ }^{17,20}$ Raft-associated $\gamma$-secretase activity is faithfully reflected in the CSF, ${ }^{4,7}$ but not in the ISF, indicating that the two $\gamma$-secretase compartments are largely separated. A particular 
subset of ISF-linked $\gamma$-secretase, raft-nonassociated $\gamma$-secretase, undergoes the refractory modulation and actively continues to generate $A \beta 1-42$ and $A \beta 1-43$ in the ISF compartment. This results in continued $A \beta$ deposition in the ISF compartment, although the CSF-linked raft-associated $\gamma$-secretase is significantly modulated and $A \beta 1-42$ and A $\beta 1-43$ levels may be extremely low in the CSF (Figure 5).

Nishimura et $\mathrm{al}^{21}$ have reported that a CHAPSO-solubilized $\gamma$-secretase from aged cynomolgus monkey brains showed a decrease of $\mathrm{A} \beta 40$ generation, but not $\mathrm{A} \beta 42$ generation. These aged monkey brains deposited large amounts of $\mathrm{A} \beta 42$, as do $\mathrm{AD}$ patients. These results are consistent with our observation of CHAPSO-solubilized raft-nonassociated $\gamma$-secretase activity measured by an in vitro assay (Figure 4). Although A $\beta 40$ generation decreased, $A \beta 40$ accumulation has increased in human brains with SP stages $\mathrm{B}$ and $\mathrm{C}$ and $\mathrm{AD}$ (Figure $2 \mathrm{~B}$ ). A reasonable explanation for this contradiction is that $A \beta 38$ and $\mathrm{A} \beta 40$ are accumulated predominantly in leptomeningeal blood vessels, and their aggregates have lower insolubility (Supplemental Figure S1). We found that the accumulation of $A \beta 1-38$ and $A \beta 1-41$ in the leptomeningeal blood vessels occurred in the cognitive normal brains at first and followed accumulation of $A \beta 1-36$ to $A \beta 1-40$ in the leptomeningeal blood vessels in $\mathrm{AD} .^{22} \mathrm{~A} \beta$ drains through the cerebrovascular basement membrane from the CSF to the lymphatic node with perivascular drainage pathway. ${ }^{23,24}$ In $\mathrm{AD}$ subjects, the levels of $\mathrm{A} \beta 1-38$ and $\mathrm{A} \beta 1-40$ in the CSF increased compared with cognitively normal subjects. ${ }^{4}$ These generated $A \beta s$ accumulate in the leptomeningeal vessels, and it might have occurred cerebral amyloid angiopathy (CAA). ${ }^{16,18,25}$ Thus, $A \beta 38$ and $A \beta 40$ seem to increase in the AD brains.

In addition, we assessed raft-associated and raftnonassociated $\gamma$-secretase activity using alcadein- $\alpha$, which is known as one substrate of $\gamma$-secretase. ${ }^{26}$ The generated species ratio of $\mathrm{p} 3$-Alc- $\alpha 35 / \mathrm{p} 3$-Alc- $\alpha 38$ is prone to increase in the raft-associated $\gamma$-secretase from $A D$ rather than normal controls (data not shown). The altered $\gamma$-secretase activity is reflected in the results of an in vitro assay using another substrate. Currently, we do not know the mechanisms behind the altered activity of both the raft-associated $\gamma$-secretase and the raft-nonassociated $\gamma$-secretase in the amyloid-bearing brains. Both $\gamma$-secretases exist in the plasma membrane and are surrounded by lipid bilayers. A change of the lipid composition in the AD brains compared with control brains could alter both $\gamma$-secretase activities in the plasma membrane. ${ }^{27}$ These lipid compositions (eg, cholesterol, phosphatidylcholine, and phosphatidylserine) are known to modify $\gamma$-secretase activity drastically. ${ }^{28-31}$ Previously, Dotti and colleagues ${ }^{29}$ showed that cholesterol might affect $\gamma$-secretase activity in neuronal cells, but they and we also showed that cholesterol loss does not affect $\gamma$-secretase activity in nonneuronal cells. ${ }^{29,32}$ Thus, these lipid compositions are especially sensitive factors for the $\gamma$-secretase activity in the brain. Lipid alteration might be the reason that raft-associated $\gamma$-secretase actively generates $\mathrm{A} \beta 1-38$ and $\mathrm{A} \beta 1-40$ into the CSF. On the other hand, raft-nonassociated $\gamma$-secretase actively generates $A \beta 1-42$ and A $\beta 1-43$ in the ISF compartment (Figure 5). The vast major fraction of $\gamma$-secretase is found in the raft-associated $\gamma$-secretase, but a minor fraction is found in the raft-nonassociated $\gamma$-secretase. Thus, it might take a few decades to form SPs in the brain because these deposited $A \beta s$ are generated by raft-nonassociated $\gamma$-secretase in the brain, which is only a minor $\gamma$-secretase fraction.

Another unexpected result of this study was that GSM-1 showed only a small modulation toward $\mathrm{AD}$ raftnonassociated $\gamma$-secretase, which has been refractory modulated rather than cognitively normal brain. Previously, we showed that GSM-1 had limited modulation efficacy in AD raft-associated $\gamma$-secretase ${ }^{7}$ because raft-associated $\gamma$-secretase in AD brains already had been modulated to generate actively $\mathrm{A} \beta 38$ and $\mathrm{A} \beta 40,{ }^{4,7}$ Thus, we need more strong $\gamma$-secretase modulators to decrease the $A \beta 42$ levels in the ISF compartment of amyloid-bearing brains, which might be involved in the parenchymal $\mathrm{A} \beta$ deposition.

\section{Acknowledgment}

We thank Dr. Bart De Strooper (VIB Center for the Biology of Disease, KU Leuven, Belgium, and UK Dementia Research Institute, University College London) for helpful suggestions and discussions.

\section{Supplemental Data}

Supplemental material for this article can be found at http://doi.org/10.1016/j.ajpath.2020.02.009.

\section{References}

1. Takami M, Nagashima $\mathrm{Y}$, Sano $\mathrm{Y}$, Ishihara S, MorishimaKawashima M, Funamoto S, Ihara Y: $\gamma$-Secretase: successive tripeptide and tetrapeptide release from the transmembrane domain of $\beta$ carboxyl terminal fragment. J Neurosci 2009, 29:13042-13052

2. Matsumura N, Takami M, Okochi M, Wada-Kakuda S, Fujiwara H, Tagami S, Funamoto S, Ihara Y, Morishima-Kawashima M: $\gamma$-Secretase associated with lipid rafts: multiple interactive pathways in the stepwise processing of $\beta$-carboxyl terminal fragment. J Biol Chem 2014, 289:5109-5121

3. Okochi M, Tagami S, Yanagida K, Takami M, Kodama TS, Mori K Nakayama T, Ihara Y, Takeda M: $\gamma$-Secretase modulators and presenilin 1 mutants act differently on presenilin $/ \gamma$-secretase function to cleave $A \beta 42$ and $A \beta 43$. Cell Rep 2013, 3:42-51

4. Kakuda N, Shoji M, Arai H, Furukawa K, Ikeuchi T, Akazawa K, Takami M, Hatsuta H, Murayama S, Hashimoto Y, Miyajima M, Arai H, Nagashima Y, Yamaguchi H, Kuwano R, Nagaike K, Ihara Y; Japanese Alzheimer's Disease Neuroimaging Initiative: Altered $\gamma$ secretase activity in mild cognitive impairment and Alzheimer's disease. EMBO Mol Med 2012, 4:344-352

5. Klunk WE, Engler H, Nordberg A, Wang Y, Blomqvist G, Holt DP, Bergström M, Savitcheva I, Huang GF, Estrada S, Ausén B, Debnath ML, Barletta J, Price JC, Sandell J, Lopresti BJ, Wall A, Koivisto P, Antoni G, Mathis CA, Långström B: Imaging brain 
amyloid in Alzheimer's disease with Pittsburgh compound-B. Ann Neurol 2004, 55:306-319

6. Mintun MA, Larossa GN, Sheline YI, Dence CS, Lee SY, Mach RH, Klunk WE, Mathis CA, DeKosky ST, Morris JC: $\left[{ }^{11} \mathrm{C}\right] \mathrm{PIB}$ in a nondemented population: potential antecedent marker of Alzheimer disease. Neurology 2006, 67:446-452

7. Kakuda N, Akazawa K, Hatsuta H, Murayama S, Ihara Y; Japanese Alzheimer's Disease Neuroimaging Initiative: Suspected limited efficacy of $\gamma$-secretase modulators. Neurobiol Aging 2013, 34:1101-1104

8. Braak H, Braak E: Neuropathological stageing of Alzheimer-related changes. Acta Neuropathol 1991, 82:239-259

9. Braak H, Alafuzoff I, Arzberger T, Kretzschmar H, Del Tredici K: Staging of Alzheimer disease-associated neurofibrillary pathology using paraffin sections and immunocytochemistry. Acta Neuropathol 2006, 112:389-404

10. Hughes CP, Berg L, Danziger WL, Coben LA, Martin RL: A new clinical scale for the staging of dementia. Br J Psychiatry 1982, 140: $566-572$

11. Yamaguchi H, Sugihara S, Ogawa A, Oshima N, Ihara Y: Alzheimer $\beta$ amyloid deposition enhanced by apoE $\varepsilon 4$ gene precedes neurofibrillary pathology in the frontal association cortex of nondemented senior subjects. J Neuropathol Exp Neurol 2001, 60:731-739

12. Kakuda N, Funamoto S, Yagishita S, Takami M, Osawa S, Dohmae N, Ihara Y: Equimolar production of amyloid beta-protein and amyloid precursor protein intracellular domain from $\beta$-carboxyl-terminal fragment by $\gamma$-secretase. J Biol Chem 2006, 281:14776-14786

13. Acx H, Chávez-Gutiérrez L, Serneels L, Lismont S, Benurwar M, Elad N, De Strooper B: Signature A $\beta$ profiles are produced by different $\gamma$-secretase complexes. J Biol Chem 2013, 289:4346-4355

14. Serneels L, Van Biervliet J, Craessaerts K, Dejaegere T, Horré K, Van Houtvin T, Esselmann H, Paul S, Schäfer MK, Berezovska O, Hyman BT, Sprangers B, Sciot R, Moons L, Jucker M, Yang Z, May PC, Karran E, Wiltfang J, D'Hooge R, De Strooper B: $\gamma$-Secretase heterogeneity in the Aph1 subunit: relevance for Alzheimer's disease. Science 2009, 324:639-642

15. Hardy J, Selkoe DJ: The amyloid hypothesis of Alzheimer's disease: progress and problems on the road to therapeutics. Science 2001, 297 : $353-356$

16. Mawuenyega KG, Sigurdson W, Ovod V, Munsell L, Kasten T, Morris JC, Yarasheski KE, Bateman RJ: Decreased clearance of CNS beta-amyloid in Alzheimer's disease. Science 2010, 330:1774

17. Weller RO: How well does the CSF inform upon pathology in the brain in Creutzfeldt-Jakob and Alzheimer's diseases? J Pathol 2001, 194:1-3

18. Zhang ET, Inman CB, Weller RO: Interrelationships of the pia mater and the perivascular (Virchow-Robin) spaces in the human cerebrum. J Anat 1990, 170:111-123

19. Weller RO, Subash M, Preston SD, Mazanti I, Carare RO: Perivascular drainage of amyloid- $\beta$ peptides from the brain and its failure in cerebral amyloid angiopathy and Alzheimer's disease. Brain Pathol 2008, $18: 253-266$
20. Zekonyte J, Sakai K, Nicoll JA, Weller RO, Carare RO: Quantification of molecular interactions between ApoE, amyloid-beta $(A \beta)$ and laminin: relevance to accumulation of $A \beta$ in Alzheimer's disease. Biochim Biophys Acta 2016, 1862:1047-1053

21. Nishimura M, Nakamura S, Kimura N, Liu L, Suzuki T, Tooyama I: Age-related modulation of $\gamma$-secretase activity in non-human primate brains. J Neurochem 2012, 123:21-28

22. Kakuda N, Miyasaka T, Iwasaki N, Nirasawa T, Wada-Kakuda S, Takahashi-Fujigasaki J, Murayama S, Ihara Y, Ikegawa M: Distinct deposition of amyloid- $\beta$ species in brains with Alzheimer's disease pathology visualized with MALDI imaging mass spectrometry. Acta Neuropathol Commun 2017, 5:73

23. Ma Q, Ineichen BV, Detmar M, Proulx ST: Outflow of cerebrospinal fluid is predominantly through lymphatic vessels and is reduced in aged mice. Nat Commun 2017, 8:1434

24. Morris AW, Sharp MM, Albargothy NJ, Fernandes R, Hawkes CA, Verma A, Weller RO, Carare RO: Vascular basement membranes as pathways for the passage of fluid into and out of the brain. Acta Neuropathol 2016, 131:725-736

25. Selkoe DJ: Clearing the brain's amyloid cobwebs. Neuron 2013, 32 $177-180$

26. Omori C, Kaneko M, Nakajima E, Akatsu H, Waragai M, Maeda M, Morishima-Kawashima M, Saito Y, Nakaya T, Taru H, Yamamoto T, Asada T, Hata S, Suzuki T; Japanese Alzheimer's Disease Neuroimaging Initiative: Increased levels of plasma p3-alc $\alpha 35$, a major fragment of Alcadein $\alpha$ by $\gamma$-secretase cleavage, in Alzheimer's disease. J Alzheimers Dis 2014, 39: $861-870$

27. Fabelo N, Martín V, Marín R, Moreno D, Ferrer I, Díaz M: Altered lipid composition in cortical lipid rafts occurs at early stages of sporadic Alzheimer's disease and facilitates APP/BACE1 interactions. Neurobiol Aging 2014, 35:1801-1812

28. Osenkowski P, Ye W, Wang R, Wolfe MS, Selkoe DJ: Direct and potent regulation of gamma-secretase by its lipid microenvironment. J Biol Chem 2008, 283:22529-22540

29. Abad-Rodriguez J, Ledesma MD, Craessaerts K, Perga S, Medina M, Delacourte A, Dingwall C, De Strooper B, Dotti CG: Neuronal membrane cholesterol loss enhances amyloid peptide generation. J Cell Biol 2004, 167:953-960

30. Holmes O, Paturi S, Ye W, Wolfe MS, Selkoe DJ: Effects of membrane lipids on the activity and processivity of purified $\gamma$-secretase. Biochemistry 2012, 51:3565-3575

31. Osawa S, Funamoto S, Nobuhara M, Wada-Kakuda S, Shimojo M, Yagishita S, Ihara Y: Phosphoinositides suppress gamma-secretase in both the detergent-soluble and -insoluble states. J Biol Chem 2008, 283:19283-19292

32. Wada S, Morishima-Kawashima M, Qi Y, Misono H, Shimada Y, Ohno-Iwashita Y, Ihara Y: Gamma-secretase activity is present in rafts but is not cholesterol-dependent. Biochemistry 2003, 42: 13977-13986 